\title{
Medial Patellofemoral Ligament Reconstruction
}

\author{
Malek Ghnaimat*, Mohannad Alodat*, Moohammad Aljazazi, Raed Alzabn \\ *Orthopedic Department, Royal Jordanian Medical Services, Orthopedic Specialists, Jordan
}

Address for Correspondence: Dr Malek Ghnaimat, Royal Jordanian Medical Services, Email: drmalekmg@yahoo.com

\begin{abstract}
Recurrent patellofemoral instability is a common disabling condition especially in young individuals. MPFL is the main stabilizer to lateral patellar displacement and its reconstruction will restore patellofemoral stability. Many techniques had been described to reconstruct MPFL with good results and few complications taking in consideration meticulous surgical technique and careful patient selection. We used hamstring autograft in a double bundle anatomic MPFL reconstruction technique with both ends of the graft fixed to patella through two blind tunnels by anchor and bioabsorbable interference screw fixing the graft through a blind tunnel in the femur at 30 degree flexion.
\end{abstract}

Key words: Medial patellofemoral ligament, patellofemoral instability, hamstring

\section{Introduction}

Recurrent patellofemoral instability is a common disabling condition especially in young individuals. The pathoanatomy of patellofemoral instability is multifactorial with risk factors including increased $\mathrm{Q}$ angle, ligamentous laxity, patella alta, small patella, trochlear dysplasia and external tibia torsion.

Studies have shown that the medial patellofemoral ligament (MPFL) is the main stabilizer against lateral patella dislocation and its reconstruction will regain patellofemoral stability in patients with recurrent patellar instability [1-3].

Many techniques had been described for MPFL reconstruction with high successful rate and low complications [1-6]. Conservative treatment is a good choice of treatment in acute dislocation of patella if no indication for surgery as osteochondral fragments or ligament complex disruption [7].

\section{Objective}

Our aim is to access our results in patients underwent MPFL reconstruction at The Royal Jordanian Medical Services (RJMS).

Manuscript received: $6^{\text {th }}$ March 2017

Reviewed: $14^{\text {th }}$ March 2017

Author Corrected: $20^{\text {th }}$ March 2017

Accepted for Publication: $27^{\text {th }}$ March 2017

\section{Materials and Methods}

It is a retrospective study done in the period between April 2014 and October 2015. Nine patients and ten cases were included in the study. Patients with moderate to severe patellofemoral arthritis, bony deformity, trochlea dysplasia and patella alta were excluded. Mean follow up was 4 months. Patients were assessed for pain, instability and present complications. Pre reconstruction arthroscopy, hamstring tendon harvested, two blind horizontal medial patellar tunnel used and femoral tunnel on the medial femoral condyle done under image.

Surgical Technique- The anatomic double blind end tunnels technique is used in reconstructing the MPFL. The graft used is the autogenous semitendinosis. Both ends of the graft are sutured at their ends to a $10 \mathrm{~mm}$ length with measuring the diameter of the ends and also the doubled graft.

A $2 \mathrm{~cm}$ incision is made between the superomedial border and the center of patella edge and two guide pins inserted, one $3 \mathrm{~mm}$ distal to the proximal medial corner of the patella and the second wire is inserted $15-20 \mathrm{~mm}$ distal to the first hole followed by over drilling by $4 \mathrm{~mm}$ reamer to a depth of $20 \mathrm{~mm}$. The ends of the graft are passed through the tunnels with fixation of the ends by anchor $4.5 \mathrm{~mm}$ and the suture ends passed to the lateral 
aspect of the patella and tied together. The anchor sutures tied to the graft.

The space between the capsule and the vastus medialis bluntly dissected and a loop is passed. The fluoroscopy is used for identifying the femoral tunnel, which will be $1 \mathrm{~mm}$ anterior to the posterior cortex of the femur, proximal to the posterior point of blumensaat line and $2.5 \mathrm{~mm}$ lower to the initial of the medial femoral condyle on lateral view. Reaming to $1 \mathrm{~mm}$ more than the measured diameter of the doubled graft. The graf ist passed and fixed by a bioabsorbable screw with tensioning at 30 degree flexion of the knee with the patella at the lateral femoral condyle edge.

Partial weight bearing for two weeks, early motion immediately to reach full motion at the end of 6 weeks and full activity by 3 months

\section{Results}

Eight patients were male and one was female who underwent bilateral MPFL reconstruction. Mean age was 28 years (20-32), with redislocation times at least was three and all primary dislocations were post traumatic results. Two patients complained of anterior knee pain with no instability but had wasted quadriceps muscle. One patient had limited flexion solved by manipulation under anesthesia. No redislocation was encountered.

\section{Discussion}

The medial patellofemoral ligament (MPFL) is the main stabilizer structure of the patella and preventing lateral patellar translation during early knee flexion. [9]. Tear of the MPFL occurs after patellar dislocation in more than $90 \%$ of the cases.

Many techniques for MPFL reconstruction have been described with different graft options and graft fixation methods. Mostly the hamstrings autograft is used, fixed at the femoral side with an interference screw and at the patellar side, either single tunnel or dual tunnels, by or suture anchors [1-6]. Most of the MPFL reconstruction procedures are using double bundle graft with two blind patellar tunnels to mimic the broad insertion of MPFL and to have optimum stability $[1-3,5,6]$ as we did to our patients. Graft fixation at the patella was by anchor suture, end bottom or just passing the graft via transverse or longitudinal tunnels [2,3]. Anatomic femoral tunnel placement is crucial, as we have done, since femoral tunnel malpositioning will lead to flexionextension lag, maltracking patella or failed procedure $[4,6]$. Tensioning of the graft is usually at 20-30degree of flexion [2,4-5]. Although a study by Sven Ostermeier et al, showed that flexion angle had little loading of the medial patellofemoral ligament autograft, which would support early mobilization of patients after surgery [9].

The complications after MPFL reconstruction are the result of technical error and associated with the specific technique used. Complications include restricted range Of knee motion (mainly loss of flexion), patellar fractures, patellar instability and patellofemoral arthritis [7]. We had two patients (20\%) continued complaining of anterior knee pain with no instability and it is reported in a study that some patients will continue to have positive apprehension test [1].

A study by John J Matheus reported 7 patients with loss of flexion and stressed on careful patient selection,we had only one case and improving [3-4]. No redislocation has been encountered in many studied and different procedures [1,3,4-6].

No patella fracture encounterd in our study, Seven Schifzadeh stressed on not using implants in fixing graft to patella which can predispose to fracture [5].

\section{Conclusion}

MPFL reconstruction is a safe and effective procedure for patellar instability if done in a meticulous way with good patient selection.

Disclosure- Their was no financial support from any company or organization.

\section{Funding: Nil, Conflict of interest: None Permission of IRB: Yes}

\section{References}

1. Mrs Krishana Kumara,Sankarram Renganthan, Clement JJoseph et al. Medial Patellofemoral reconstruction for patellar instability, Indian Journal of orthopedics 9-2014; 48 (5):501-5. doi: 10. 4103 /00195413.139864.

2. Michael RCarmen, Nicolar Maffuli. Medial patellofemoral ligament reconstruction: a new technique, BMC Musculoskeletal disorders, 2007; 8: 22. DOI: $10.1186 / 1471-2474-8-22$. 
3. John J. Mathews, Peter Schranz. Reconstruction of medial patellofemoral ligament using a longitudinal tunnel technique International Orthopedics 2010, 34; 1321-1325.doi:10.1007/s00264-009-0918-7.

4. Reddy K Raghuveer, Chandra B, Mishra. Recostruction of medial patellofemoral ligament for chronic instability. Indian J Orthopedics 2012;46(4) 447-454. doi: 10.4103/0019-5413.97259.

5. Seven Shfizadeh, Maurice Balke. Medial patellofemoral ligament reconstruction: A new technique for graft fixation at patella without implant. Arthroscopy Technique 2014; 3(1):e115-e117. doi: 10. 1016/j.eats.2013.09.005.

6. Alexander Golant, Tony Quach, and Jeffrey E. Rosen. Medial Patellofemoral Ligament Reconstruction With a Looped Semitendinosus Tendon, Using Knotless Anchor Fixation on the Patella and Hybrid Fixation on the Femur. Arthroscopy technique 2014;3(2):e211e216. doi: 10.1016/j.eats.2013.10.001.
7. Buchner M, Baudendistel B, Sabo D, Schmitt H. Acute traumatic primary patellar dislocation:long-term results comparing conservative and surgical treatment. Clin J Sport Med. 2005 Mar;15(2):62-6.

8. Jay N Shah,Jennifer S Howard,David C Flanigan,et al. A systemic review of complications and failures associated with medial patellofemoral ligament reconstruction for recurrent patellar dislocation. Am J Sports Med. 2012 August; 40(8): 1916-1923. doi: 10. $1177 / 0363546512442330$.

9. Sven Ostermeier, Christina Stukenborg-Colsman. In Vitro Investigation of the Effect of Medial Patellofemoral Ligament Reconstruction and Medial Tibial Tuberosity Transfer on Lateral Patellar Stability. Arthroscopy The Journal of Arthroscopic and Related Surgery 04/2006; 22(3):308-19. DOI:10.1016/j.arthro. 2005.09.024.

\section{How to cite this article?}

Malek Ghnaimat, Mohannad Alodat, Moohammad Aljazazi, Raed Alzabn. Medial Patellofemoral Ligament Reconstruction. Int J Med Res Rev 2017;5(03):371-373 doi:10.17511/ijmrr. 2017.i03.26. 